\title{
ESTUDO DE DEPOSIÇÃO DE CERA EM ÓLEOS PARAFÍNICOS
}

\author{
M. L. ALCANTARA ${ }^{1}$, S. BARRETO ${ }^{1}$, M. C. MEIRELLES ${ }^{1}$, G. B. TARANTINO ${ }^{1}$, L. M. N. \\ GOES $^{1}$, C. A. M. PIRES ${ }^{1}$, L. C. L. SANTOS ${ }^{1}$ e S. MATTEDI ${ }^{1}$ \\ ${ }^{1}$ Programa de Pós-Graduação em Engenharia Química, Universidade Federal da Bahia \\ E-mail para contato: lclsantos@ufba.br
}

RESUMO - Um dos problemas mais comuns na produção e transporte do petróleo é a deposição de parafinas. Este problema resulta em prejuízos financeiros devido a maiores gastos com manutenção e energia. Diferentes modelos termodinâmicos já foram desenvolvidos e aplicados para a determinação de alguns destes parâmetros como a composição da cera e a temperatura inicial de aparecimento de cristais (TIAC). Este trabalho tem como objetivo estudar o fenômeno da deposição de parafinas, com foco na determinação da TIAC e na composição do sólido formado e comparar modelos para a descrição destas propriedades. Foram medidas, experimentalmente, a TIAC por densimetria, a quantidade de saturados, aromáticos, resinas e asfaltenos (SARA), a composição do óleo por cromatografia gasosa e a quantidade e composição da cera. A TIAC e a quantidade e composição de cera foram calculadas através da aplicação de quatro modelos termodinâmicos a fase líquida considerando a fase sólida uma solução ideal e comparado com os resultados de óleos obtidos de seis campos.

\section{INTRODUÇÃO}

$\mathrm{Na}$ indústria do petróleo é de fundamental importância a capacidade de prever e controlar a deposição das parafinas. As deposições de parafina são barreiras presentes nas paredes dos dutos que prejudicam o escoamento do petróleo pelas linhas de fluxo, chegando em casos extremos a parar a produção. O estudo sobre este fenômento pode ser utilizado como uma ferramenta para garantir um escoamento seguro e econômico. Diferentes modelos já foram propostos e aplicados na determinação da composição da cera e da temperatura de aparecimento de cristais (TIAC). Este trabalho tem como objetivo comparar modelos para descrever o fenômeno da deposição de ceras, com foco na determinação da TIAC e na composição do sólido formado. Para complementar este objetivo e poder comparar os modelos são estudados experimentalmente o comportamento da deposição de cera em óleos de campos maduros do recôncavo baiano.

\section{MATERIAIS E MÉTODOS}

\subsection{Procedimento Experimental}

A cromatografia gasosa foi utilizada para determinar a composição do óleo e da cera. Para a caracterização do óleo e da cera foi utilizado um cromatógrafo a gás Varian (CP-3800) 
de acordo com a ASTM 2887. O cromatógrafo realiza a análise baseado na temperatura e na vazão do gás e o resultado apresentado tem um erro de $\pm 0,7 \mu \mathrm{ml} / \mathrm{min}$

A precipitação da cera foi feita utilizando uma técnica mostrada por Martos et al. (2010) e Espada et al. (2010), que foi uma modificação do método desenvolvido por Burger et al.(1981), em que a cera foi obtida através da diluição do óleo com solventes e resfriada durante $24 \mathrm{~h}$ e então, separada por filtração. Uma amostra deste sólido separado também foi analisada por cromatografia gasosa.

Outra análise efetuada no óleo foi a SARA. Este método determina a percentagem mássica de saturados, aromáticos, resinas e asfaltenos, por cromatografia líquida em coluna aberta. São coletadas amostras de saturados, aromáticos e compostos NSO (compostos que contém nitrogênio, enxofre e oxigênio). A incerteza na massa inicial de óleo e nas frações é igual a $\pm 0,0001 \mathrm{~g}$.

Para a determinação da TIAC utilizou-se a medida da densidade através de um densímetro digital Anton Paar DMA 4500 para fazer uma curva de densidade com o intervalo de temperatura de $60^{\circ} \mathrm{C}$ a $12^{\circ} \mathrm{C}$. O valor da densidade tem incerteza de $\pm 0,00001 \mathrm{~g} / \mathrm{cm}^{3}$ e o ensaio da densidade foi realizado de acordo com a Martos D4052-11.

\subsection{Cálculo da TIAC e Composição de Cera}

Para realizar o cálculo da TIAC foi considerado que a cera é formada apenas por $n$ alcanos cristalizando dos hidrocarbonetos fluidos como cristais ortorrômbicos em solução sólida. Essa consideração foi baseada em evidências experimentais mostradas na literatura (COUTINHO et al., 1996; COUTINHO, 1998).

A descrição termodinâmica da formação da cera é realizada utilizando uma equação geral para equilíbrio sólido-líquido que relaciona a composição com a não idealidade das fases e com propriedades termofísicas de componentes puros, que é a Equação 1.

$K=\frac{\gamma^{L}}{\gamma^{S}} \cdot \exp \left\{\frac{\Delta H^{f u s}}{R T_{f u s}} \cdot\left(\frac{T_{f u s}}{T}-1\right)+\frac{\Delta H^{t r}}{R T_{t r}} \cdot\left(\frac{T_{t r}}{T}-1\right)+\frac{\Delta C p}{R} \cdot\left[\log \left(\frac{T_{f u s}}{T}\right)-\frac{T_{f u s}}{T}+1\right]\right\}$

Essas propriedades são calculadas em relação ao número de carbono dos componentes $(C n)$. A temperatura de fusão $\left(T_{\text {fus }}\right)$ e entalpia de fusão $\left(\Delta H^{f u s}\right)$ foram estimados segundo as Equações 2 e 3 (MARANO e HOLDER, 1997). Já a diferença do calor específico $(\Delta C p)$ foi estimados de acordo com a Equação 4 (ERICSON et al., 1993; WON, 1986; LIRAGALEANA et al., 1996).

$$
\begin{aligned}
& T_{f u s}=421,63-1936412 \cdot \exp \left[-7,8945(C n-1)^{0,07194}\right] \\
& \Delta H^{f u s}=0,00355 \cdot C n^{3}-0,2376 \cdot C n^{2}+7,400 \cdot C n-34,814 \\
& \Delta C p=0,2557 \cdot C n-4,4245 \cdot 10^{-4} C n \cdot T_{f u s}
\end{aligned}
$$

A temperatura de transição $\left(T_{t r}\right)$ e a entalpia de transição $\left(\Delta H^{t r}\right)$ foram calculadas segundo Marano e Holder (1997) pelas Equações 5 e 6. 


$$
\begin{aligned}
& \Delta H^{t r}=37791 C n-126540-\Delta H^{f u s} \\
& T_{t r}=420,42-134784 \cdot \exp \left[-4,344(C n+6,592)^{0,14627}\right]
\end{aligned}
$$

Para o cálculo do equilíbrio sólido-líquido foi considerado a fase sólida como ideal e para a fase líquida foi realizada uma comparação com modelos de coeficiente de atividades. Devido à consideração de que apenas precipitam n-parafinas, a composição inicial do óleo, obtida através da cromatografia, foi corrigida utilizando a Equação 7. Essa aproximação foi feita para aproximar a quantidade de $n$-alcanos da realidade.

$$
Z_{i}=\text { Ztot }_{i} \cdot s a t
$$

$\mathrm{O}$ termo $Z_{\text {tot }}$ se refere à composição total, somando todas as frações. O termo sat se refere a percentagem de saturados presentes em cada óleo determinado através da análise SARA.

Então, para a resolução do algoritmo, as equações tradicionais de Rachford-Rice (ELLIOT e LIRA, 2012) foram utilizadas no sistema multifásico descrito acima e através do método de Newton-Raphson para cálculos iterativos obtém-se os resultados requeridos. Foram feitos dois tipos de cálculos, primeiro resolve-se o sistema para determinação da TIAC e composição da primeira cera a precipitar. Logo, resolve-se a equação para a temperatura de deposição da cera $(-20 \mathrm{C})$ e determina-se a quantidade e a composição da cera depositada. Quatro modelos termodinâmicos foram aplicados para resolver a Equação 1: Uniquac preditivo, Unifac, Flory de volume livre e Wilson preditivo.

Uniquac preditivo: Para o cálculo do coeficiente de atividade através do UNIQUAC Preditivo (COUTINHO et al., 2000) foi feita a consideração que os termos $r$ e $q$ são calculados através do número de carbonos contidos nos componentes, através das Equações 8 e 9.

$$
\begin{aligned}
& r_{i}=0,0148 \cdot C n+0,00996 \\
& q_{i}=0,0185 \cdot C n+0,0211
\end{aligned}
$$

Além disso, no cálculo do parâmetro empírico $\tau_{i j}$, utiliza-se o termo $\lambda$, que representa a energia de interação binária e é relacionado com o calor de sublimação do componente puro como calculado pela Equação 10.

$$
\lambda_{i i}=-\frac{2}{10}\left(\Delta H^{s u b}-R T\right)
$$

Sendo $\lambda_{\mathrm{ij}}=\lambda_{\mathrm{jj}}$ onde, $j$ é o $n$-alcano mais curto do par $i j$.

A entalpia de sublimação foi calculada pela Equação 11.

$$
\Delta H^{s u b}=\Delta H^{v a p}+\Delta H^{f u s}+\Delta H^{t r}
$$

A entalpia de vaporização foi calculada pelo método PERT2 segundo Morgan e Kobayashi (1993). 
Unifac: Já o UNIFAC modificado foi mostrado por Larsen et al. (1987) e utiliza a Equação 12 para o cálculo do parâmetro $\phi$.

$$
\phi_{\mathrm{i}}=\frac{\mathrm{x}_{\mathrm{i}} \mathrm{r}_{\mathrm{i}}^{2 / 3}}{\sum_{\mathrm{j}} \mathrm{x}_{\mathrm{j}} \mathrm{r}_{\mathrm{j}}^{2 / 3}}
$$

O parâmetro de volume $r_{i}$ foi calculado segundo a Equação 13, apresentada por Coutinho (1998).

$$
\mathrm{r}_{\mathrm{i}}=0,1 C n_{i}+0,0672
$$

Flory de volume livre: O coeficiente de atividade pelo modelo de Flory de Volume Livre (COUTINHO et al., 1995) considera as diferenças de tamanho e as contribuições de volume livre, sendo calculado através das Equações 14 e 15.

$$
\begin{aligned}
& \gamma_{\mathrm{i}}^{\mathrm{L}}=\exp \left[\ln \left(\frac{\varphi_{\mathrm{i}}^{\mathrm{L}}}{\mathrm{x}_{\mathrm{i}}^{\mathrm{L}}}\right)+1-\frac{\varphi_{\mathrm{i}}^{\mathrm{L}}}{\mathrm{x}_{\mathrm{i}}^{\mathrm{L}}}\right] \\
& \varphi_{\mathrm{i}}^{\mathrm{L}}=\frac{\mathrm{x}_{\mathrm{i}}^{\mathrm{L}} \cdot\left(\mathrm{Vm}_{\mathrm{i}}^{1 / 3}-\mathrm{Vw}_{\mathrm{i}}^{1 / 3}\right)^{3,3}}{\sum \mathrm{x}_{\mathrm{i}}^{\mathrm{L}} \cdot\left(\mathrm{Vm}_{\mathrm{i}}^{1 / 3}-\mathrm{Vw}_{\mathrm{i}}^{1 / 3}\right)^{3,3}}
\end{aligned}
$$

Wilson preditivo: A Equação 16 foi demonstrada por Hiranuma (1974), que ajustou a equação de Wilson para a utilização em sistemas multicomponente.

$$
\ln \gamma_{\mathrm{i}}=1-\ln \sum_{\mathrm{i}} \mathrm{x}_{\mathrm{j}} \Lambda_{\mathrm{ij}}-\sum_{\mathrm{k}} \frac{\mathrm{x}_{\mathrm{k}} \Lambda_{\mathrm{ki}}}{\sum_{\mathrm{j}} \mathrm{x}_{\mathrm{j}} \Lambda_{\mathrm{kj}}}
$$

Sendo, a energia de interação contida no cálculo do parâmetro $\Lambda_{\mathrm{ki}}$ calculadas seguindo a Equação 10. E considerando também que $\lambda_{\mathrm{ij}}=\lambda_{\mathrm{jj}}$ onde, $j$ é o $n$-alcano mais curto do par $i j$.

\section{RESULTADOS}

A percentagem de saturados, aromáticos e compostos NSO está na Tabela 1 e como pode ser observado, os resultados confirmam a característica parafínica dos óleos do Recôncavo Baiano. A composição de cera que é depositada e a TIAC dos óleos foram estimadas através da metodologia descrita na seção 2 e os desvios relativos dos valores calculados em relação aos experimentais podem ser observados nas Tabelas 2 e 3.

Através da Tabela 2 podemos perceber que, no geral, os modelos conseguiram prever bem a TIAC, pois os erros foram baixos, exceto para o petróleo 5, que teve uma maior discrepância. Isso pode ser devido ao fato do petróleo 5 ter apresentado impurezas, que foram observadas na realização dos experimentos e devem ter interferido nos resultados. 
Tabela 1 - SARA dos óleos

\begin{tabular}{cccc}
\hline Petróleo & \% SAT & \% ARO & \% NSO \\
\hline 1 & 74,53 & 15,29 & 10,18 \\
2 & 66,76 & 13,64 & 19,59 \\
3 & 77,98 & 13,26 & 8,76 \\
4 & 70,00 & 18,27 & 11,73 \\
5 & 85,67 & 11,76 & 2,57 \\
6 & 88,67 & 10,00 & 1,33 \\
\hline
\end{tabular}

Tabela 2 - Desvios relativos da TIAC

\begin{tabular}{ccccc}
\hline Óleo & $\begin{array}{c}\text { UNIQUAC } \\
\text { Preditivo }\end{array}$ & UNIFAC & $\begin{array}{c}\text { Flory de Volume } \\
\text { Livre }\end{array}$ & $\begin{array}{c}\text { Wilson } \\
\text { Preditivo }\end{array}$ \\
\hline 1 & $7,72 \%$ & $3,63 \%$ & $5,69 \%$ & $16,57 \%$ \\
2 & $7,73 \%$ & $3,29 \%$ & $3,38 \%$ & $13,80 \%$ \\
3 & $7,70 \%$ & $5,51 \%$ & $5,65 \%$ & $16,25 \%$ \\
4 & $7,80 \%$ & $3,65 \%$ & $5,68 \%$ & $15,34 \%$ \\
5 & $13,41 \%$ & $10,21 \%$ & $12,14 \%$ & $19,09 \%$ \\
6 & $7,68 \%$ & $3,93 \%$ & $5,96 \%$ & $14,62 \%$ \\
\hline
\end{tabular}

Analisando os desvios em relação aos modelos apresentados, pode ser observado que o modelo UNIFAC apresenta os menores erros relativos, se mostrando o melhor modelo para descrever o equilíbrio sólido-líquido quando o petróleo estiver na temperatura de aparecimento inicial dos cristais.

O que se pode perceber da Tabela 3 é que os desvios relativos encontrados para o percentual de cera foram altos e no caso do modelo Wilson Preditivo não foi possível encontrar valores com significado físico para o percentual de cera nos óleos 2 a 6 . A tendência do modelo foi a de superestimar os valores, inclusive extrapolando o limite físico, mostrando que esse modelo não se adequou ao perfil dos petróleos parafínicos.

As medidas experimentais estão sempre menores do que as calculadas, principalmente as do óleo 5, que como mencionado, apresentou impurezas, influenciando diretamente no cálculo do percentual de cera existente na amostra, por isso apresentou altos desvios.

Analisando os resultados da porcentagem de cera encontrada nos óleos de uma maneira geral, pode-se perceber que o modelo Flory de volume livre apresentou os menores desvios relativos em todas as amostras. E além de ter apresentado os melhores resultados para o percentual de cera, este modelo também apresentou bons resultados na predição da TIAC, mostrando valores próximos ao melhor modelo apresentado, que foi UNIFAC. Por isso, se tiver que escolher um modelo que melhor descreva o comportamento do óleo no geral, ou seja, incluindo a TIAC e a porcentagem de cera, o modelo Flory de Volume Livre é o melhor modelo, que também já foi aceito em vários trabalhos (COUTINHO et al., 1995; COUTINHO, 1998; COUTINHO et al., 2006). 
Tabela 3 - Desvios relativos do percentual de Cera

\begin{tabular}{ccccc}
\hline Óleo & $\begin{array}{c}\text { UNIQUAC } \\
\text { Preditivo }\end{array}$ & UNIFAC & $\begin{array}{c}\text { Flory de Volume } \\
\text { Livre }\end{array}$ & $\begin{array}{c}\text { Wilson } \\
\text { Preditivo }\end{array}$ \\
\hline 1 & $44,96 \%$ & $18,25 \%$ & $16,49 \%$ & $90,39 \%$ \\
2 & $26,25 \%$ & $17,94 \%$ & $17,47 \%$ & - \\
3 & $36,09 \%$ & $23,60 \%$ & $22,60 \%$ & - \\
4 & $91,17 \%$ & $71,44 \%$ & $69,68 \%$ & - \\
5 & $153,11 \%$ & $139,83 \%$ & $138,35 \%$ & - \\
6 & $29,91 \%$ & $18,12 \%$ & $17,09 \%$ & - \\
\hline
\end{tabular}

Os perfis para os diversos óleos são semelhantes, para exemplificar pode-se observar na Figura 1 o perfil da composição da cera do petróleo 1.

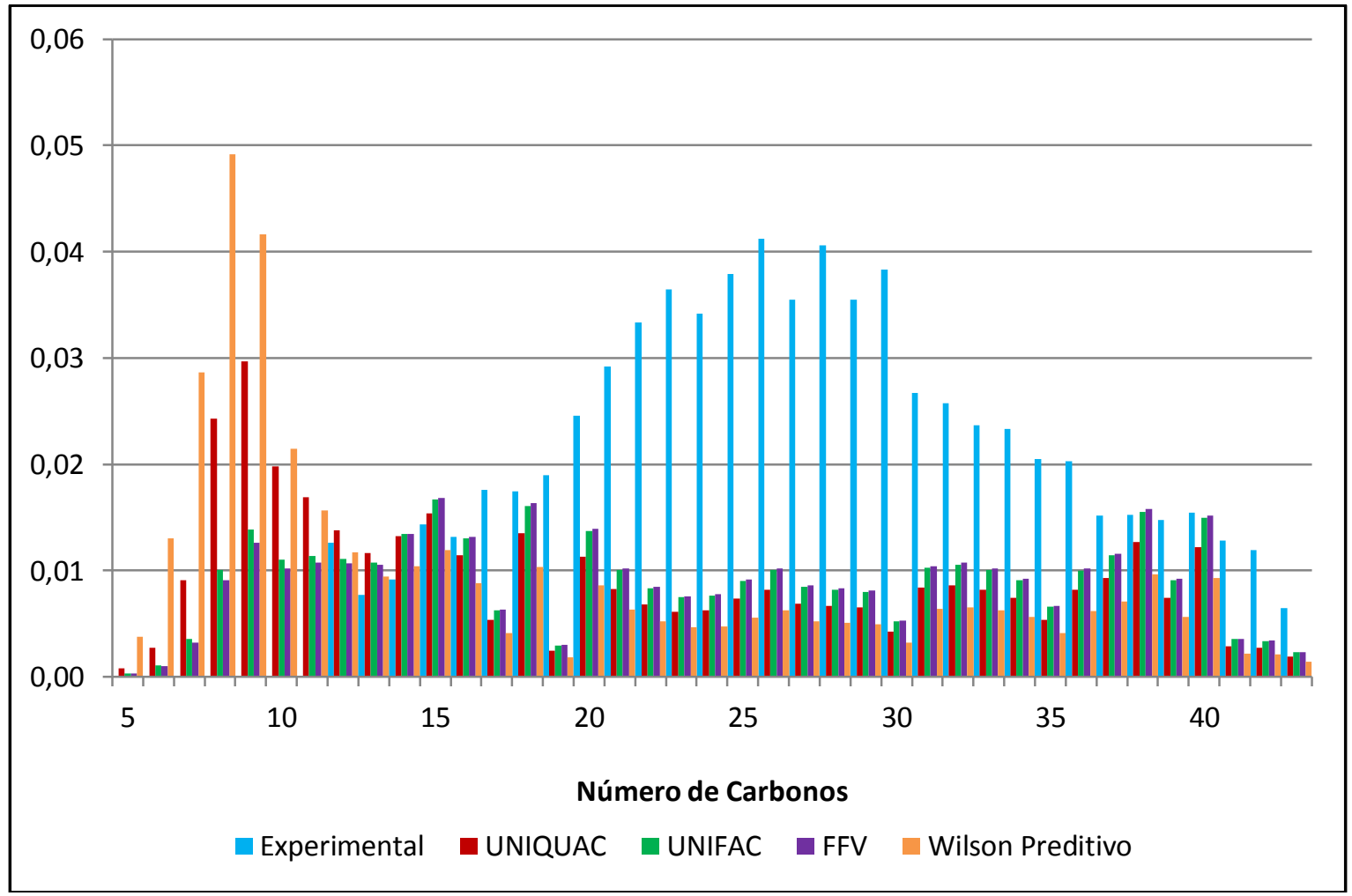

Figura 1-Composição da cera e do óleo1

Ao analisar a Figura 1, nota-se que os perfis encontrados pelos modelos escolhidos no trabalho foram semelhantes entre si, sendo que o UNIQUAC Preditivo e o Wilson Preditivo apresentaram uma maior predição em compostos com baixo número de carbono, até o C12. Isso acontece devido à consideração de solução sólida, onde todos os compostos precipitam, por isso os modelos superestimam esses compostos, já que na realidade, por observação experimental, a cera começa a existir com compostos acima de 12 carbonos. 
Além desta consideração levar a modelagem a erros, existe também o efeito da aproximação do coeficiente de atividade do sólido ser considerado igual a um. Na Figura 1 é analisada especificamente a composição da cera, que é a parte sólida da mistura, que foi considerada como ideal. Essa consideração pode ter levado a erros elevados na predição tanto da porcentagem de cera precipitada quanto da composição desta cera. Por isso, a melhor alternativa quando o foco da informação for a porcentagem de cera precipitada é não considerar a fase sólida como ideal e utilizar os coeficientes de atividade específico para $\mathrm{o}$ cálculo de sólidos para descobrir um valor mais próximo ao real.

\section{CONCLUSÃO}

Os estudos de deposição de cera são fundamentais para a compreensão do comportamento do escoamento e precipitação do petróleo, em especial em campos maduros. Neste trabalho foi determinado características de 6 óleos do Recôncavo Baiano. Foram determinadas a TIAC, a composição dos óleos e das ceras e uma análise SARA dos óleos. Foi calculada a TIAC e a composição do sólido a $-20^{\circ} \mathrm{C}$ considerando a não idealidade do líquido e considerando a fase sólida ideal. Concluiu-se que comparando os modelos utilizados o que apresentou melhores resultados na TIAC foi o UNIFAC. Já para o cálculo do percentual de cera os resultados não foram bons, refletindo a consideração da idealidade da fase sólida. Assim, para um resultado satisfatório das características do sólido é necessário o cálculo do equilíbrio termodinâmico considerando a fase sólida como não ideal.

\section{AGRADECIMENTOS}

Os autores agradecem a Petróleo Brasileiro, S. BARRETO agradecem a CAPES. G. B. M. L. ALCANTARA e TARANTINO agradecem ao CNPq.

\section{REFERENCIAS}

ASTM 2887, "Standard Test Method for Boiling Range Distribution of Petroleum Fractions by Gas Chromatography”, ASTM International, 2008.

ASTM D 4052-11, "Standard Test Method for Density, Relative Density, and API Gravity of Liquids by Digital Density Meter”, ASTM International, 2002.

BURGER, E. D.; PERKINS, T. K.; STRIEGLER, J. H. Studies of Wax Deposition in the Trans Alaska Pipeline. Society of Petroleum Engineers of AIME,v. 33 p. 1075-1086, 1981.

COUTINHO, J. A. P.; ANDERSEN, S. I.; STENBY, E. H. Evaluation of Activity Coefficient Models in Prediction of Alkane Solid-Liquid Equilibria. Fluid Phase Equilibria, v.103, p. 23-39, 1995.

COUTinho, J. A. P.; KNUdSEn, K.; Andersen, S. I.; STEnBY, E. H. A Local Composition Model for Paraffinic Solid Solutions. Chemical Engineering Science, v.51, n.12, p. 3273-3282, 1996. 


\section{9 a 22 de outubro de 2014 \\ Florianópolis/SC}

COUTINHO, J. A. P. Predictive UNIQUAC: A New Model for the Description of Multiphase Solid-Liquid Equilibria in Complex Hydrocarbon Mixtures. Ind. Eng. Chem. Res., v. 37, p.4870-4875, 1998.

COUTINHO, J. A. P.,DAUPHIN, C., DARIDON, J. L. Measurements and modeling of wax formation in diesel fuels. Fuel, v.79 p. 607-616, 2000.

COUTINHO, J. A. P.; MIRANTE, F.; PAUly, J. A New Predictive UNIQUAC for Modeling of Wax Formation in Hydrocarbon Fluids. Fluid Phase Equilibria, v.247, p.8$17,2006$.

ELLIOT, J.R., LIRA, C.T. Introductory Chemical Engineering Thermodynamics, New York: Editora Prentice Hall; 2. ed., 2012.

ESPADA, J. J., COUTINHO, J. A. P., PEÑA, J. L. Evaluation of Methods for the Extraction and Characterization of Waxes from Crude Oils. Energy \& Fuels, v.24, p.1837-1843, 2010.

LARSEN, B. L., RASMUSSEN, P., FREDENSLUND, A. A modified UNIFAC groupcontribution model for prediction of phase equilibria and heats of mixing, Ind. Eng. Chem. Res., v. 26, p. 2274-2286, 1987.

LIRA-GALEANA, C., FIROOZABADI, A., PRAUSNITZ, J.M. Thermodynamics of wax precipitation in petroleum mixtures. AIChE Journal, v.42, p.239-248, 1996.

MARANO, J.J.; HOLDER, G.D. General Equations for Correlating the Thermo-physical Properties of n- Paraffins, n-Olefins and other Homologous Series. 2. Asymptotic Behavior Correlations for PVT Properties. Ind. Eng. Chem. Res.,v.36, p. 1887-1894, 1895.

MARTOS, C., COTO, B., ESPADA, J. J., ROBUSTILlO, M. D., PEÑA J. L., MERINOGARCIA, D. Characterization of Brazilian Crude Oil Samples to improve the predictions of Wax Precipitation in Flow Assurance Problems. Energy and Fuels. v.24 p. 2221-2226, 2010.

MORGAN, D., KOBAYASHI, R. Extension of Pitzer CSP models for vapor pressures and heats of vaporization to long-chain hydrocarbons. Fluid Phase Equilibria, v. 94, p. 51-87, 1993.

WON, K.W. Thermodynamics for solid solution-liquid-vapor equilibria: wax phase formation from heavy hydrocarbon mixtures, Fluid Phase Equilibria, v.30, p.265-279, 1986. 